\title{
Corrigendum
}

\section{Corrigendum to sequence determinants for DNA packaging specificity in the $S$. aureus Pathogenicity Island SaPI1 [Plasmid 71 (2014) 8-15]}

\author{
Joana C. Bento ${ }^{\mathrm{a}, \mathrm{b}}$, Kristin D. Lane ${ }^{\mathrm{a}}$, Erik K. Read ${ }^{\mathrm{c}}$, Nuno Cerca ${ }^{\mathrm{b}}$, Gail E. Christie ${ }^{\mathrm{a}, *}$
}

a Department of Microbiology and Immunology, Virginia Commonwealth University School of Medicine, PO Box 980678, Richmond, VA 23298-0678, USA

b Centre of Biological Engineering, IBB - Institute for Biotechnology and Bioengineering, University of Minho, Campus de Gualtar, Braga 4710-057, Portugal

${ }^{\mathrm{c}}$ Division of Monoclonal Antibodies, Center for Drug Evaluation and Research, Food and Drug Administration, 10903 New Hampshire Avenue, Silver Spring, MD 20993, USA

The authors regret inaccuracies in Figure 4 resulting from reversing the transduction titers for pJCB12 and pJCB13. A corrected replacement version of this figure is provided.

The authors would like to apologize for any inconvenience caused.

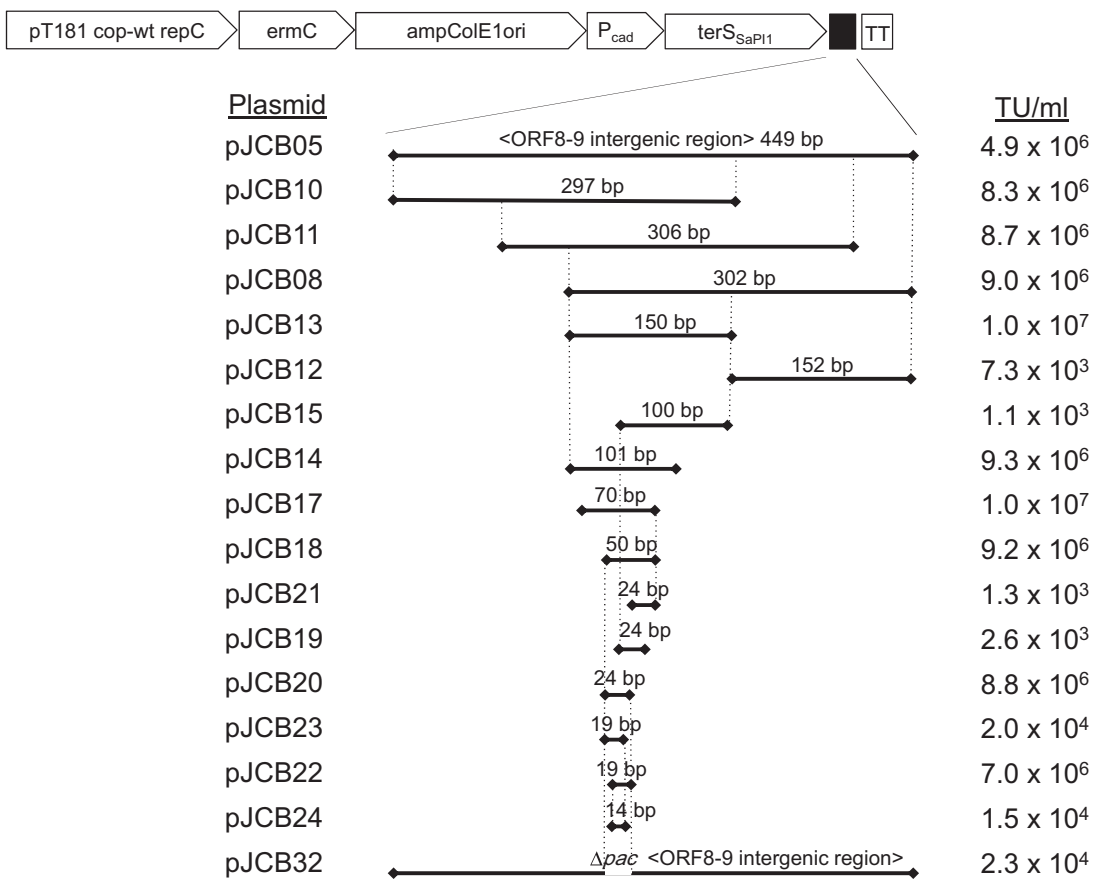

doi of original article: 10.1016/j.plasmid.2013.12.001

* Corresponding author. Department of Microbiology and Immunology,

Virginia Commonwealth University School of Medicine, PO Box 980678, Richmond, VA 23298-0678, USA. Fax: 804 828-9946.

E-mail address: christie@vcu.edu (G.E. Christie). 